\title{
Status and inspiration on the development of the air monitoring system ANITA for European Space Agency
}

\author{
Zhengyang Yuan ${ }^{1 *}$, Jiayong Zhou ${ }^{1}$, Cuncun Qian ${ }^{2}$, Shizhong Wang ${ }^{1}$, Juntao Zhao ${ }^{1}$, Hongqi Shi ${ }^{1}$ \\ ${ }^{1}$ Wuhan Second Ship Design and Research Institute, 430205, Wuhan, China \\ ${ }^{2}$ Huazhong University of Science and Technology, Audit Office, 430074, Wuhan, China
}

\begin{abstract}
This paper describes the technical characteristics and application status of ANITAII (Analysing Interferometer for Ambient Air), an atmospheric monitoring system currently used by ESA (European Space Agency), reveals the characteristics and advantages of FTIR (Fourier transform infrared spectroscopy) technology in multi-component gas monitoring, and analyzes the application prospect of FTIR technology in the field of environmental monitoring.
\end{abstract}

\section{Introduction}

ANITA is a permanent continuous trace gas monitoring system on the International Space Station (ISS), which is jointly developed by ESA and NASA (National Aeronautics and Space Administration)[1]. Based on FTIR technology, the air analyser can simultaneously monitor 32 trace gases with ppm or sub-ppm detection limits[2]. The system can measure with a time resolution in the order of minutes, which allows to trace the dynamic changes in the concentrations of trace gases in the ISS for the first time. In the view of the successful application of FTIR technology in ANITA project, this paper analyzes the application prospect of FTIR technology in the field of environmental monitoring.

\section{Basic principles of FTIR}

FTIR had been evaluated by ESA as the best technology for continuous monitoring of cabin air, and its technology maturity had been successfully confirmed in a large number of Breadboard (BB) experiments and blind sample tests of NASA[3].

The principles of infrared spectrum measurement is shown in Fig.1.

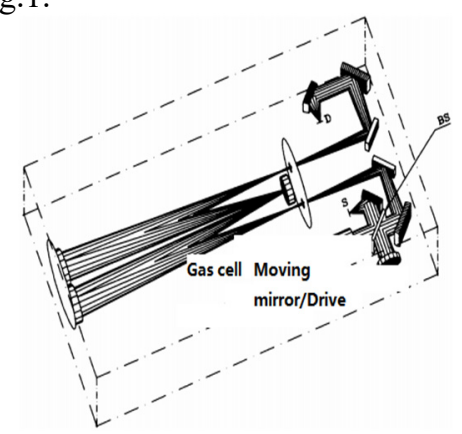

Fig1. Operation principle of the FTIR measurement system, $\mathrm{S}$ means light source.

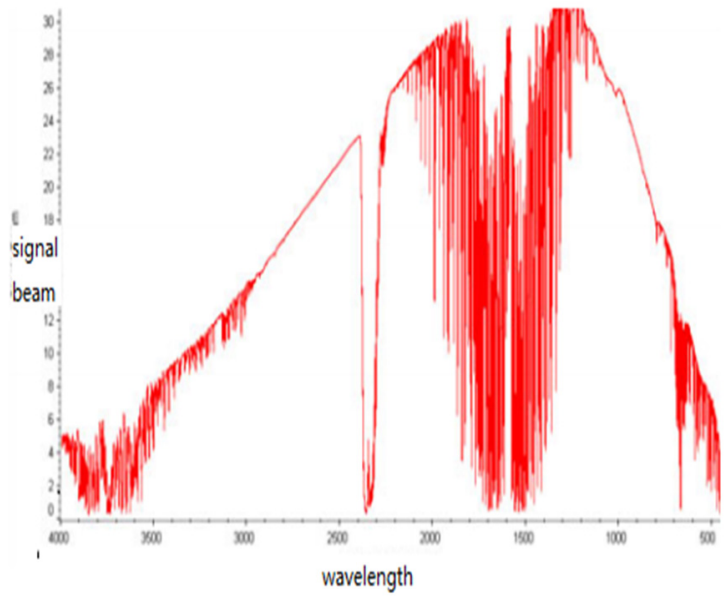

Fig2. Typical FTIR spectrum detected by ANITA.

The modulated infrared radiation from the broadband light source is guided through the air chamber where the air sample (target gas) exists, and then the system detects the radiation, and transforms the measured signal by the mathematical Fourier transform method, finally obtaining the characteristic spectrum as shown in Fig. 2. The wave number range is selected among $600 \sim 3500 \mathrm{~cm}-1$, in order to monitor the characteristic absorption line of each gas component in parallel. The position and details of absorption band are used for qualitative identification of gas, and the absorption depth is used for corresponding quantitative analysis.

Infrared (IR) technology has several advantages, including high wavelength stability, high optical resolution and high efficiency, which make IR spectrometer suitable for gas measurement. At the same time, the complex situation in multi-gas analysis requires complex data analysis. Therefore, by the time ESA developed the monitoring technology based on FTIR principle in the TGM (Trace Gas Monitoring) scheme, it 
also developed the data analysis technology based on PLS (partial least square) method.

The FTIR modulator is the core of a FTIR spectrometer, which represents Michelson Interferometer as shown in Fig.3. It has a beam splitter, a fixed and a movable mirror. The incoming ray signal is modulated by the movable mirror, and the interference figure produced by coherent ray is detected by the detector. By applying the mathematical Fourier transform method to the detection signal which is a function of time, the measurement signal is converted into a spectrogram for performing the corresponding gas analysis. The microvibration injected into the system during the measurement process will cause the moving mirror to tilt and move, thus reducing the system performance.

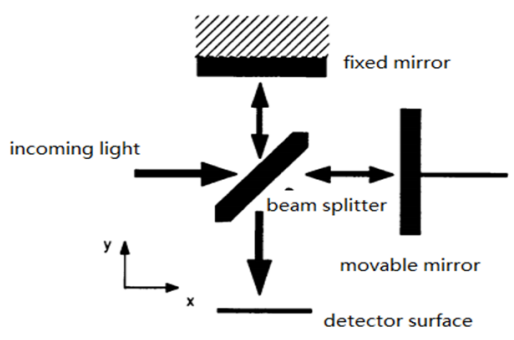

Fig3. FTIR modulator.spectral ghosts

\section{ANITA \& its technical characteristics}

In Fig.4, ANITA I measurement is shown on the left, and ANITA II on the right.
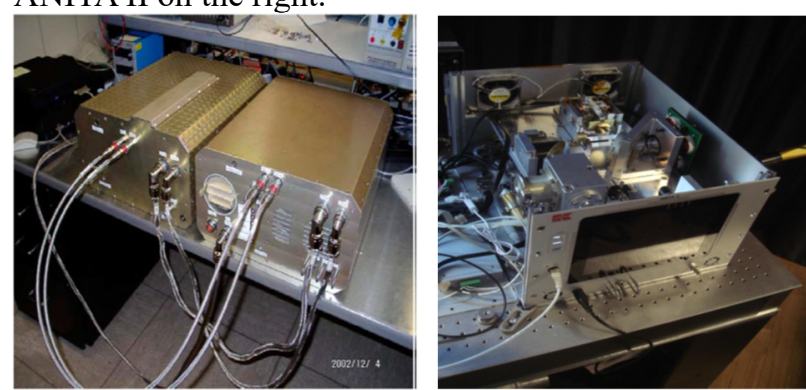

Fig4. ANITA I measurement units (left) and ANITA II measurement units (right).

ANITA I program came from ESA's TGM scheme, it began in 1991, and was launched in August in 2007. It has been running on the ISS for 11 months and produced outstanding data sets[4].

ANITA can automatically detect and quantify various gases in complex mixtures, even if the background gas such as $\mathrm{H} 2 \mathrm{O}$ and $\mathrm{CO} 2$ vary greatly. The total signal-tonoise ratio (SNR) in the absorption spectrum depends on the SNR of the foreground and background spectra. In each measurement, the absorption spectrum is calculated by a foreground spectrum (the spectrum measured on the air sample) and a background spectrum as a reference measurement. Based on the stability of ANITA instrument and the effective baseline drift compensation in analysis software, the background spectrum is set to be updated every 12 hours in order to minimize the cycle time. As a compromise between the need for short cycle time on the hand and the allocation of time for signal integration and air sample update on the other hand, the cycle time of each measurement includes one minute of signal integration time and two minutes of response time[5].

For long-term health problems, such as cancer risk caused by air pollutants, system cycle is not important. However, for possible short-term problems, such as direct danger of crew poisoning, detection of leakage or overheating of equipment, and inspection of effect of corrective measures, low cycle time may be necessary. The cycle time is set to 5 minutes.

In the laboratory, we usually measure the background spectrum in a gas chamber filled with pure N2. Provided the gas chamber strong enough, high vacuum can be used instead of N2. ANITA relies on another solution, that is, obtaining low pressure through internal pump for sampling. The pump can reduce the air chamber pressure to $1500 \mathrm{~Pa}$ (about $1.5 \%$ of the measured pressure). This process provides a clean background[6].

ANITAI can monitor 32 gases online at the same time with sub-ppm accuracy, the gas varieties are as follows in Tab.2[7].

Table1. Gas scenario from ANITAI

\begin{tabular}{|c|c|c|c|}
\hline \multicolumn{2}{|r|}{ Compound } & \multirow{2}{*}{ CUCL(ppm) } & \multirow{2}{*}{$\begin{array}{l}\text { Estimated } \\
\text { detection } \\
\text { limit }(\mathrm{ppm})\end{array}$} \\
\hline No. & Name & & \\
\hline 1 & methanol & 70 & 0.10 \\
\hline 2 & ethanol & 30 & 0.3 \\
\hline 3 & 2-propanol & 30 & 0.5 \\
\hline 4 & 1-butanol & 50 & 1.0 \\
\hline 5 & formaldehyde & 5 & 0.10 \\
\hline 6 & acetaldehyde & 30 & 0.4 \\
\hline 7 & propionaldehyde & 50 & 0.4 \\
\hline 8 & butyraldehyde & 40 & 0.3 \\
\hline 9 & toluene & 6 & 0.9 \\
\hline 10 & meta-xylene & 15 & 0.4 \\
\hline 11 & ortho-xylene & 6 & 0.4 \\
\hline 12 & para-xylene & 20 & 0.2 \\
\hline 13 & ethyl benzene & 20 & 0.9 \\
\hline 14 & Ethyl acetate & 5 & 0.3 \\
\hline 15 & n-butyl acetate & 4 & 0.2 \\
\hline 16 & dichloro methane & 12 & 0.3 \\
\hline 17 & Freon 11 & 2 & 0.013 \\
\hline
\end{tabular}




\begin{tabular}{|c|c|c|c|}
\hline 18 & Freon 12 & 1 & 0.04 \\
\hline 19 & Halon 1301 & 1.25 & 0.05 \\
\hline 20 & Freon 113 & 3 & 0.05 \\
\hline 21 & perfluoro propane & 100 & 0.03 \\
\hline 22 & hexane (n-) & 12 & 0.3 \\
\hline 23 & acetone & 10 & 0.4 \\
\hline 24 & 2-butanone & 30 & 0.3 \\
\hline 25 & $\begin{array}{l}\text { hexamethyl cyclo- } \\
\text { trisiloxane }\end{array}$ & 2 & 0.003 \\
\hline 26 & $\begin{array}{l}\text { octamethyl cyclo- } \\
\text { tetrasiloxane }\end{array}$ & 1.2 & 0.003 \\
\hline 27 & $\begin{array}{l}\text { Decamethyl cyclo- } \\
\text { pentasiloxane }\end{array}$ & 1 & 0.012 \\
\hline 28 & ammonia & 4 & 0.06 \\
\hline 29 & carbon monoxide & 10 & 0.05 \\
\hline 30 & methane & 500 & - \\
\hline 31 & carbon dioxide & 10000 & - \\
\hline 32 & water & 25028 & - \\
\hline
\end{tabular}

Essential characteristics of the calibration methods include[8]:

- Synthetic calibration exploiting the advantages of the FTIR technique

- Control of optical spectral interference (the main problem)

- Baseline drift compensation (solves a major problem)

- Optimal noise handling utilizing information from numerous spectral data points

- Control of possible optical saturation effects (too high spectral absorption)

- Simple, fast, and fully automatic runtime calculations

- Easy adaptations by recalibration, ever after deployment in space (without astronaut involvement)

The concentration of each gas refers to 25 and standard atmospheric pressure, that is, $101.3 \mathrm{kPa}$. The temperature and pressure of air samples will lead to the change of infrared spectral line structure. Tests show that ANITA is robust enough to handle relatively small temperature changes (for example \pm 2 ) and moderate pressure drops $(10 \mathrm{kPa})$ with high quality. Large changes may lead to slight or significant quality degradation, but will not lead to failure.

During the ANITA I mission, strong artifacts were observed in many spectra. In the "wrong" spectral region, these artifacts appear as signals similar to $\mathrm{CO} 2$ and $\mathrm{H} 2 \mathrm{O}$, that is why they are called "ghosts" [9]. Fig.5 shows spectral ghosts after eliminating all real features from the spectrum. This effect was investigated after ANITA I mission. The researchers specially developed a mathematical model and successfully simulated the results quite close to reality. The result shows that there is vibration acting on the modulator of ANITA I instrument, and finally found that the hardware with problems is the movable mirror (or its whole base), fixed mirror and/or beam splitter[10].

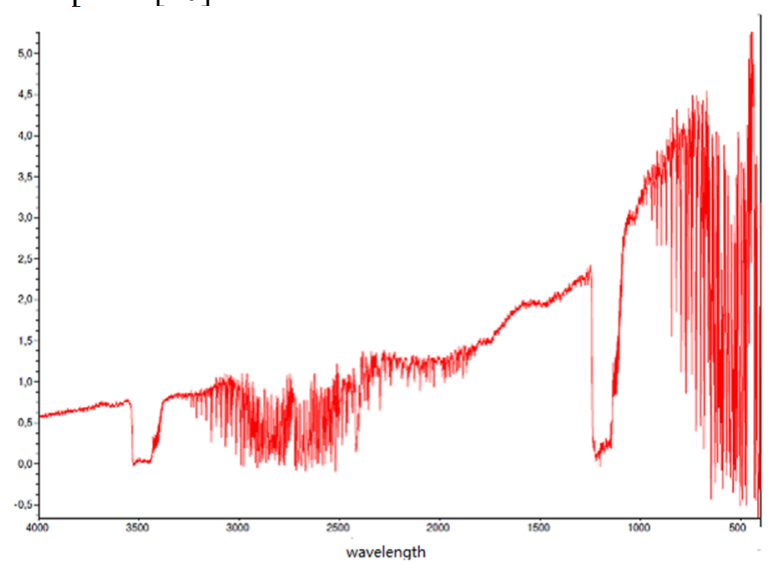

Fig5. Spectral ghosts.

ANITA II is mainly characterized by miniaturization, and its performance is mainly to eliminate the hardwarerelated interference and instability as well as increase the detection accuracy. Tab. 2 compares the instrument characteristics of ANITA I and ANITA II [11].

Table2. Comparison of ANITA I and ANITA II instrument

\begin{tabular}{|c|c|c|}
\hline System property & ANITA I & ANITA II \\
\hline Mass & $54 \mathrm{~kg}$ & $27 \mathrm{~kg}$ \\
\hline Elements & $\begin{array}{c}\text { Two mid-deck } \\
\text { locker inserts, } \\
\text { connecting gas } \\
\text { tubes and data } \\
\text { cables, laptop, } \\
\text { breakout board }\end{array}$ & $\begin{array}{c}\text { One mid-deck } \\
\text { locker insert }\end{array}$ \\
\hline Power & 150W (peak) & $100 \mathrm{~W}$ (peak) \\
\hline $\begin{array}{c}\text { Sensitivity } \\
\text { Response time } \\
\text { full set of data } \\
\text { with 33 gases and } \\
\text { potential outliers) }\end{array}$ & $3-5$ minutes & $3-5$ minutes \\
\hline
\end{tabular}

The sensitivity of each gas is an independent value, which to a great extent depends on the gas itself. ANITA II is usually an order of magnitude more sensitive than ANITA I in the operation of the ISS.

Base on the experience of ANITAI and the previous research of ANITAII, a new modulator driving device has 
been developed, which greatly reduces the microvibration of modulator and the displacement tilt of mirror.
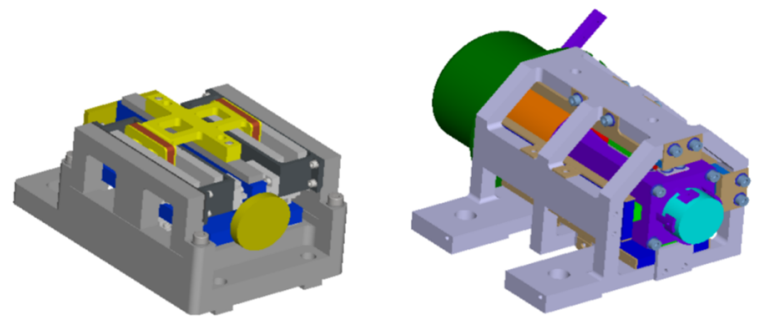

Fig6. Design sketch of the new modulator (left) compared to previous version.

For the purpose to reduce the required volume, mass and power consumption, it is necessary to develop a laser diode system with stable temperature to replace the heavy $\mathrm{He}-\mathrm{Ne}$ laser. The requirement of frequency stability of diode laser leads to the development and manufacture of customized analog electronic board suitable for DFB laser diode. The total mass and volume of the circuit board are about $10 \%$ of the mass and volume of the He-Ne laser[12].

\section{Prospect}

ANITAII may be the most outstanding achievement in the application of FTIR technology. It is small in size, light in weight, stable in operation and powerful in function, and can monitor 32 gases online at the same time with subppm accuracy. In addition to short-term manned spacecraft and medium-to-long term manned space stations, ESA will also develop ANITAIII to apply in projects such as Moon or Mars base, which will greatly promote the development of human spaceflight. At the same time, ANITAII can also be applied to other confined spaces such as submersibles, large-scale warehousing and cold storage, and underground hedging facilities to ensure the safety and reliability of scientific research or hedging environment.

The successful application of ANITA will also strengthen our confidence in FTIR technology. In terms of the breadth of gas monitoring, FTIR technology has advantages that other monitoring technologies do not have, such as TDLAS and DOAS. In the field of complex gas monitoring, such as atmospheric environment monitoring and toxic harmful gases in industrial areas, FTIR technology can continue to shine brilliantly.

\section{References}

1. T. Stuffler, D. Kampf, H. Mosebach, et al. The flight experiment ANITA-a high performance air analyser for manned space cabins, Acta Astronautica 55 (2004) 573-579.

2. T. Stuffler, H. Mosebach, D. Kampf, et al. ANITA and ANITAII Optimised Analysers for Advanced Air Quality Monitoring in Crewed Space Craft. SAE 2004-01-2265, ICES 2004, Colorado.

3. T. Stuffler, D. Kampf, H. Mosebach, et al. ANITA- the optimised spacecraft cabin air monitor on its way to orbit, ICES 2003, Vancouver.
4. T. Stuffler, H. Mosebach, D. Kampf, et al. The Air Quality Monitor ANITA- Going into Operation on the International Space Station, SAE 2007-01-3148, ICES 2007, Chicago.

5. T. Stuffler, H. Mosebach, D. Kampf, et al. Advanced ISS Air Monitoring-The ANITA and ANITA 2 Missions. SAE International 2009-01-2523, ICES 2009, Savannah.

6. T. Stuffler, H. Mosebach, D. Kampf, et al. Evaluation of ANITA Air Monitoring on the International Space Station. SAE 2009-02-2520, ICES 2009, Savannah.

7. T. Stuffler, H. Mosebach, D. Kampf, et al. The ANITA Air Monitoring Programme and Instrumentation-ISS and Other Applications. SAE 2006-01-2176, ICES 2006, Virginia.

8. T. Stuffler, H. Mosebach, D. Kampf, et al. ANITA2The Future High Performance Air Monitor for ISS Trace Gas Contamination and Gas Concentration Dynamics Monitoring, AIAA 2010-6093, ICES 2010.

9. Honne A., Schumann-Olsen H., Kampf D., et al. ANITA air monitoring on the International Space Station Part2: Air Analyses, SAE 2008-01-2043, ICES 2009.

10. T. Stuffler, S. Gutruf, H. Mosebach, et al. Status Report on ANITA2-The European Programme on High Performance Air Monitoring of ISS Trace Gas Contamination and Gas Concentration Dynamics. AIAA 2011-5211, ICES 2011, Oregon.

11. T. Stuffler, S. Gutruf, K. Nader, et al. Progress in ANITA2, the Upcoming High Performance ISS Air Monitor for Continuous In-Orbit Operation, ICES2014-68, Arizona.

12. T. Stuffler, P. Hofmann, V. Klein, et al. Progrress in the Development of the Advanced ISS Air Monitor ANITA2-BB results and alternative ways for an implementation of the program. ICES-2015-91, Washington. 